\title{
TOWARDS SUPERCAPACITORS IN SPACE APPLICATIONS
}

\author{
Brandon Buergler ${ }^{(1)}$, Bertrand Faure ${ }^{(2)}$, David Latif ${ }^{(3)}$, Lukas Diblik ${ }^{(3)}$, Petr Vasina ${ }^{(3)}$, Valéry Gineste ${ }^{(2)}$,Marek \\ Simcak $^{(4)}$ \\ (1) ESTEC, Keplerlaan 1, PO Box 299, 2200 AG Noordwijk, The Netherlands, Email: brandon.buergler@esa.int \\ (2) AIRBUS DEFENCE \& SPACE - Space Systems, 31 rue des Cosmonautes - Z.I. du Palays, 31402 Toulouse Cedex 4, \\ France,Email: bertrand.faure@astrium.eads.net \\ (3) EGGO Space, EGGO Space s.r.o., Dvořákova 328, 56301 Lanškroun, Czech Republic, Email: vasinap@eggo.cz \\ (4) CSRC, spol. s.r.o., Janska 12, 60200 Brno, Czech Republic, Email: marek.simcak@csrc.cz
}

\begin{abstract}
Supercapacitors offer a wide range of applications for space flight. The aim of this activity was to pursue life tests on commercial off the shelf (COTS) supercapacitors from different manufacturers, to evaluate their performance after long term vacuum exposure and to investigate balancing designs for the use of these cells in banks of supercapacitors (BOSC). This study enabled to select the most suitable part for space applications and to confirm the design rules at unit level and deratings at component level, which need to be applied. All those complementary results have paved the way to the on-going activities related to Nesscap 10F qualification and associated modular Bank Of Supercapacitors development for space applications.
\end{abstract}

\section{INTRODUCTION}

Supercapacitors also called electrochemical double layer capacitors are energy storage devices that store electric energy in the double layer between a high surface area electrode and an electrolyte [1]. As compared to batteries no chemical reactions should occur at the electrodes. Thus, with exception of parasitic electrochemical reactions, the storage of energy in supercapacitors is a purely physical phenomenon [2]. The use of supercapacitors on spacecrafts and launchers can be manifold. Currently, applications as power system stabilisation and energy storage for pyrotechnic mechanisms and electro-mechanical thrust vector actuation are envisaged [3]. The supercapacitor cells must be capable of withstanding the harsh environments present for space applications. The application of supercapacitors in micro-satellites is also an option, which has been studied by Shimizu and Underwood [4]. The advantage of supercapacitors is their extremely high power density of several tens of $\mathrm{kW} / \mathrm{kg}$ and their capability of operating at temperatures down to $-40^{\circ} \mathrm{C}$. The main challenge of this technology is the limited energy density, which is in the range of $5 \mathrm{Wh} / \mathrm{kg}$ [5]. Due to the purely physical operation principle supercapacitors are capable of performing a large amount of cycling. Furthermore, the performance of supercapacitors with decreasing temperature (e.g. $-40^{\circ} \mathrm{C}$ ) does not deteriorate as strongly as compared to the currently used Li-ion chemistries.

The ageing of carbon based supercapacitors becomes evident by the increase of the Equivalent Series Resistance (ESR) and in parallel a decrease of capacity with lifetime. Two types of electric aging tests are usually done. On the one hand the supercapacitor is subjected to a long duration at constant voltage and somewhat increased (compared to room temperature) temperature, on the other hand the supercapacitor is electrically cycled at e.g. room temperature. In the calendaric test the aging rate has been shown to increase with increasing temperature and voltage and in the cycling tests the capacity fade and ESR increase become more pronounced with increasing cycle number [6]. The results shown in this paper have been elaborated within an ESA led activity [7]. The aim of this work was to evaluate the application of supercapacitors in space applications and has partially been presented in [8]. This paper is a continuation of the work in terms of life test duration and an extension to the balancing system, which is of major importance for the lifetime of supercapacitor banks. Environmental tests, electrical characterisation and life tests were done both on single cells and on module level and these results shall be reported here. The Nesscap $10 \mathrm{~F}$ cell was selected owing to its good performance in the life tests. This paper focuses on this cell type. Not included are the modelling of supercapacitor cells electric performance and ageing, which are reported in [9]

\section{EXPERIMENTAL}

\subsection{Single Cell Tests}

The tests on three COTS supercapacitors reported in [7] and [8] were continued:

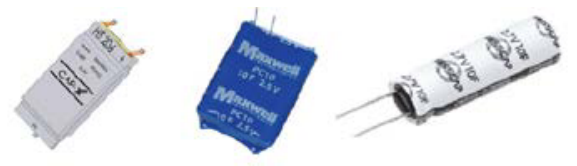




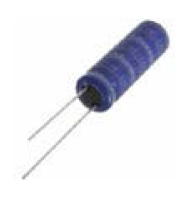

Figure 1: The four tested commercial off the shelf supercapacitor types from left to right Cap XX HS130 (2.4 F), Maxwell PC10 (10 F), Nesscap EHSR 0010C0$002 R 7$ (10 F), Maxwell BCAP0010 P270.

Cap-XX HS130 (2.4 F), Maxwell PC10 (10 F) and Nesscap 10F $(10 \mathrm{~F})$. In addition, a new COTS part from Maxwell, BCAP0010 P270 was submitted for evaluation testing. The different supercapacitor types are shown in Figure 1.

The cells were submitted to calendaric aging test at different conditions with temperatures of $-35^{\circ} \mathrm{C}, 22^{\circ} \mathrm{C}$, $45^{\circ} \mathrm{C}$ and $65^{\circ} \mathrm{C}$ and voltages of $0.6,0.8,1.0$ and 1.2 times the rated voltage (Vop). As compared to Nesscap cells, a reduced testplan with shorter durations was carried out on Maxwell cells (6000 hours). Different cycling tests with different electric profiles were also performed on the individual supercapacitors at room temperature. The results obtained with the new Maxwell BCAP0010 P270 during the 75\% discontinuous energy cycling as shown in Figure 2 shall be shown here. The results are compared to the Nesscap EHSR 0010C0002R7 cell.

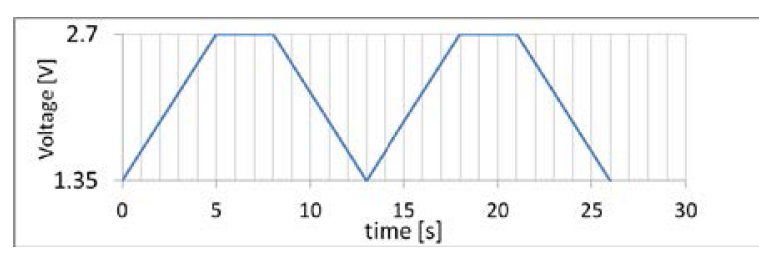

Figure 2 Cycling profile for the $75 \%$ discontinuous
energy cycling test.

Finally, 10 Nesscap parts were submitted to life test under vacuum conditions $(<10 \mathrm{mbar})$ at $0.8 \cdot \mathrm{Vop}$ at $45^{\circ} \mathrm{C}$ and under ambient pressure also at $0.8 \cdot$ Vop in a temperature range from 35 to $45^{\circ} \mathrm{C}$. This test was done to check the cells long term compatibility with space environment.

\subsection{Bank of Supercapacitor Tests}

As previously described the Nesscap cell was chosen as the baseline owing to the good performance in the tested conditions. In [7] the design and manufacturing and testing of a $15 \mathrm{~s} 2 \mathrm{p}$ bank of supercapacitors was described. The unit had in internal resistance of less than $160 \mathrm{~m} \Omega(\mathrm{DC})$ and a capacity of $1.26 \mathrm{~F}$ at beginning of life (BOL). In this paper the extension of the cycling test to more than 4 Million cycles is reported.

A further topic was to assess the dependence of the lifetime of the BOSCs on the balancing resistor. For the $15 \mathrm{~s} 2 \mathrm{p}$ BOSC each cell pair was fitted with a parallel resistor of $532 \Omega$. This allows for having balanced voltages for all the cells in the BOSC, however, it also induces a loss of efficiency in the unit. In order to study the influence of the balancing resistor, $4 \mathrm{~s} 1 \mathrm{p}$ BOSCs were manufactured by CSRC and tested at EGGO. An overview of the manufactured BOSCs is given in Table 1. Three BOSCs with different balancing factor were based on the Nesscap cell, one on the Maxwell cell and one on the Cap-XX cell. The balancing factor is defined as the ratio between balancing current at $0.80 \cdot \mathrm{Vop}$ and the leakage current of a cell at BOL. The manufactured BOSCs (Figure 3) were tested without housing.

\begin{tabular}{|c|c|c|c|c|c|}
\hline $\begin{array}{l}\text { B } \\
\text { O } \\
\text { S } \\
\text { C } \\
\mathrm{N} \\
\text { O }\end{array}$ & $\begin{array}{l}\text { Components } \\
\text { manufacturer and } \\
\text { reference }\end{array}$ & $\begin{array}{c}\text { ESR } \\
{[\Omega]}\end{array}$ & $\begin{array}{l}\text { Capac } \\
\text { ity }[\mathrm{F}]\end{array}$ & $\begin{array}{c}\text { Balancing } \\
\text { Factor }\end{array}$ & $\begin{array}{l}\text { Balancing } \\
\text { resistor } \\
{[\Omega]}\end{array}$ \\
\hline 1 & $\begin{array}{l}\text { Nesscap EHSR } \\
0010 \text { C0-002R7 }\end{array}$ & 0.078 & 2.24 & 50 & 2150 \\
\hline 2 & $\begin{array}{l}\text { Nesscap } \quad \text { EHSR } \\
0010 \text { C0-002R7 }\end{array}$ & 0.078 & 2.22 & 100 & 1000 \\
\hline 3 & $\begin{array}{lr}\text { Nesscap } & \text { EHSR } \\
0010 \text { C0-002R7 }\end{array}$ & 0.080 & 2.25 & \begin{tabular}{|l} 
No \\
balancing
\end{tabular} & $\begin{array}{l}\begin{array}{l}\text { No } \\
\text { balancing }\end{array} \\
\end{array}$ \\
\hline 6 & $\begin{array}{l}\text { Maxwell } \\
\text { BCAP0010 P270 }\end{array}$ & 0.228 & 2.48 & 100 & 681 \\
\hline 7 & Cap-XX HS130F & 0.098 & 0.56 & 100 & 3160 \\
\hline
\end{tabular}

Table 1: Overview of the BOSCs tested in the activity.

The PCB design was common for all the cells and is shown in Figure 4. The total balancing resistor per cell could be tuned by choosing the right resistor values in the two strings of two series connected resistors.

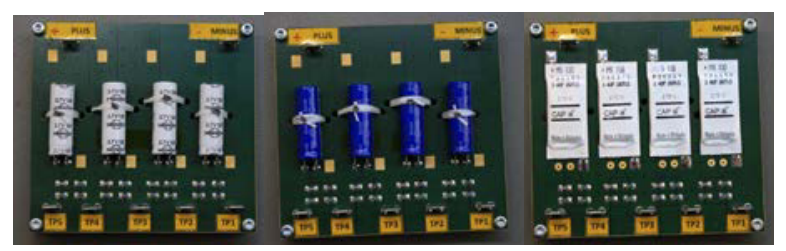

Figure 3: The manufactured banks of supercapacitors based on Nesscap (only BOSC Nr.1 shown), Maxwell and Cap-XX cells respectively. 


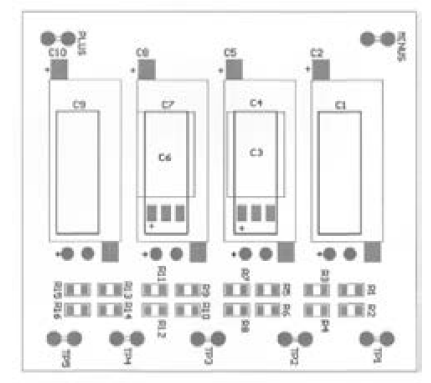

Figure 4: PCB of the 4s1p BOSCs allowing to use a single PCB design for all the studied cells and allowing flexibility in terms of balancing resistors.

The 4s1p BOSCs were initially characterised and then subjected to a cycling profile as shown in Figure 5 at $45^{\circ} \mathrm{C}$. It should be noted that no initial "balancing" of the BOSCs was done to adjust to the same level the individual cell voltages before the tests began. The capacity, ESR and leakage current were determined in regular intervals. The individual cell voltages were also measured in order to be able to check the balancing state of each BOSC.

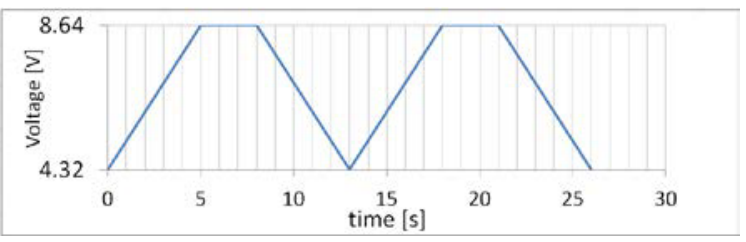

Figure 5 Cycling profile for the 4s1p BOSCs.

In the electric test profile for the BOSCs as shown in Figure 5, the maximum operation voltage was chosen to be $8.64 \mathrm{~V}$, which corresponds to $2.16 \mathrm{~V}$ (=0.8.Vop ) per cell. The cell rated voltage Vop for the Nesscap cell is $2.7 \mathrm{~V}$. Therefore using $2.16 \mathrm{~V}$ as the maximum voltage during cycling corresponds to a derating of $20 \%$, which was shown to be adequate to allow for long enough lifetimes (see cell life tests). The test profile consisted of charging the BOSC from $4.32 \mathrm{~V}$ to $8.64 \mathrm{~V}$ within 5 seconds. A current of around $2 \mathrm{~A}$ was needed at BOL for doing so. Then there was a short rest time of $3 \mathrm{~s}$ followed by a discharge to $4.32 \mathrm{~V}$. The BOSC was immediately recharged after it reached $4.32 \mathrm{~V}$.
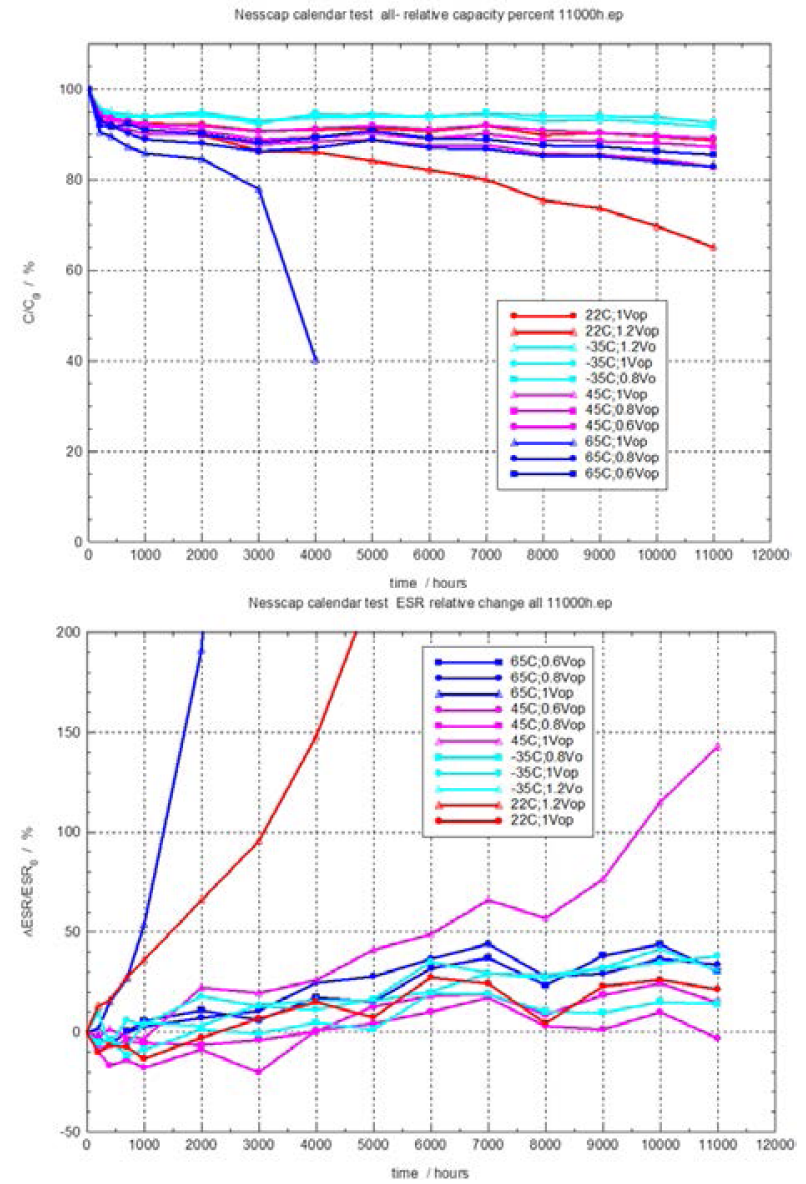

Figure 6: Capacity fade and ESR during calendaric aging of the Nesscap supercapacitors at different operating voltages and temperatures.

Thereafter the cycle was repeated. Before each intermediate check-up every 100'000 cycles of the BOSCs the individual cell voltage measurement was done. This was done by discharging the BOSC and acclimatising to $23^{\circ} \mathrm{C} \pm 2{ }^{\circ} \mathrm{C}$ for two hours. Thereafter, the BOSCs were charged to $8.64 \mathrm{~V}$ with a current of $0.8 \mathrm{~A}$. Then the individual cell voltages were measured while the BOSCs were kept at constant voltage of $8.64 \mathrm{~V}$. The capacity of the BOSCs was determined by cycling the BOSCs at $0.45 \mathrm{~A}(0.11 \mathrm{~A}$ for BOSC07) for 10 times between 0 and 10,8 V (11 V for BOSC07). The capacity was determined from the last discharge to $0 \mathrm{~V}$.

\section{RESULTS AND DISCUSSION}

\subsection{Single Cell Tests}

The calendaric ageing of the Nesscap supercapacitors is shown in Figure 6, those of Maxwell in Figure 7. The average of five individual specimens is shown per test condition. Vop corresponds to the values given in the data sheet of the cells (for Nesscap, Cap-XX and Maxwell: $2.7 \mathrm{~V}$ ). At $45^{\circ} \mathrm{C}$ and $0.8 \cdot$ Vop, which will be the expected maximum temperature for a cell in a conventional spacecraft, the Nesscap and Maxwell specimens showed capacity fade of around $10 \%$ after 
6000 hours (Figure 6). In the same conditions, the ESR increase is around $20 \%$ for Nesscap and for Maxwell.
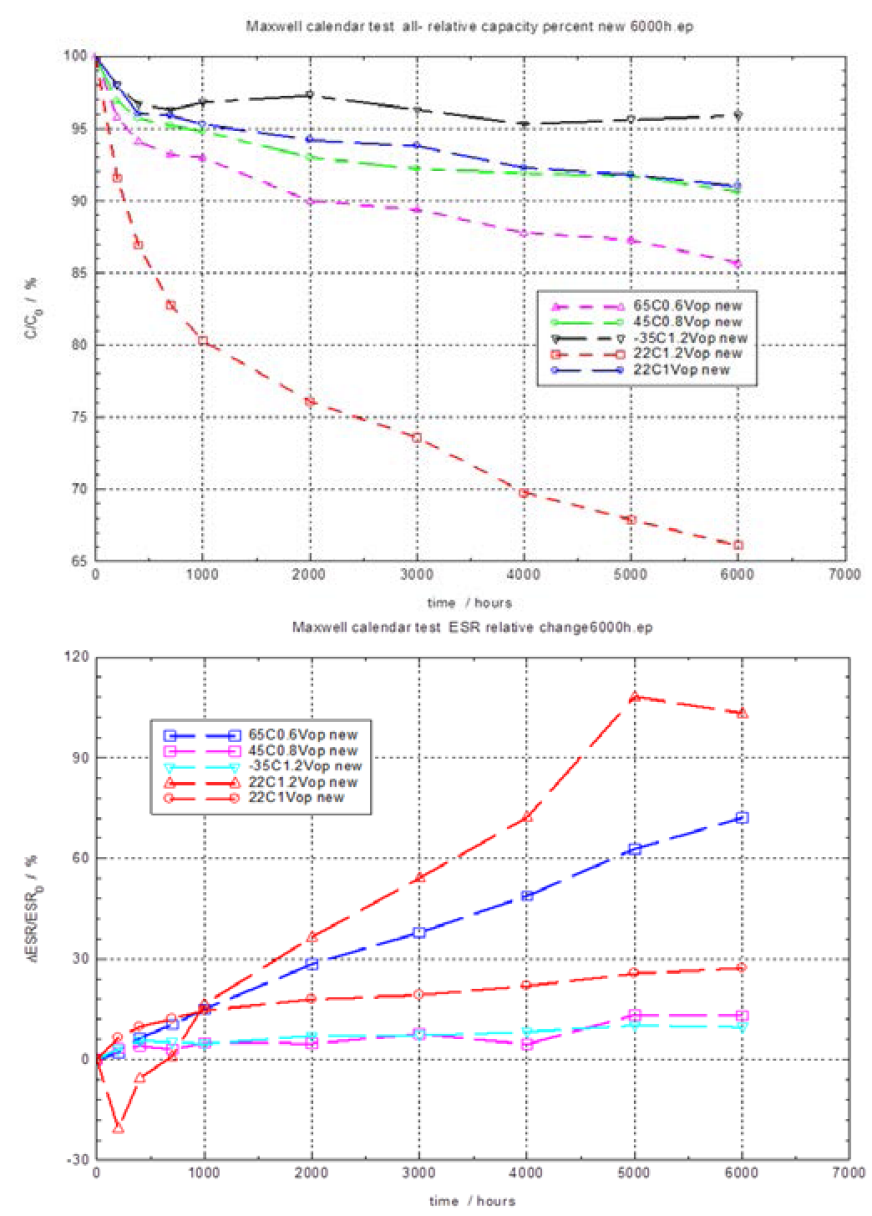

Figure 7: Capacity fade and ESR during calendaric aging of the Maxwell BCAP0010 P270 supercapacitors at different operating voltages and temperatures.

A derating of $0.85 \cdot \mathrm{Vop}$ and $45^{\circ} \mathrm{C}$ appears to be a good compromise between sufficient durability for space applications and simplified thermal management.

Cycle life test up to 800.000 cycles in continuous $100 \%$, discontinuous $75 \%$ and continuous $75 \%$ conditions were also performed on Maxwell BCAP0010 P270. The results of the $75 \%$ discontinuous cycling is shown in Figure 8 . The relative capacity losses of both cell types are similar. The evolution of ESR with cycles is more pronounced for Maxwell cells, however, after 600'000 cycles the cells have a similar relative ESR increase. It has to be noted that the DC ESR at the BOL of the Maxwell cell is about twice the value of the Nesscap cell (55 $\mathrm{m} \Omega$ vs $27 \mathrm{~m} \Omega)$.
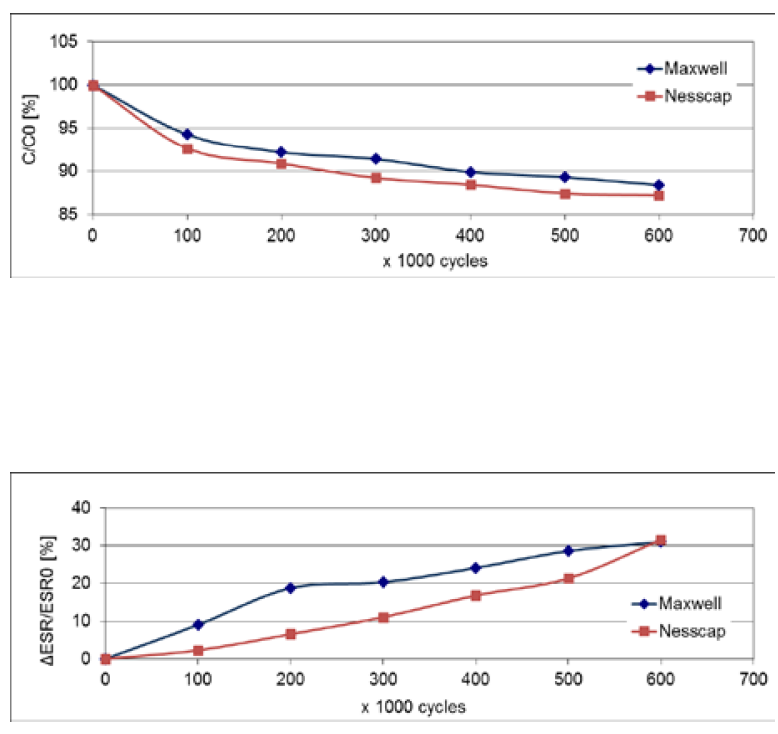

Figure 8: Capacity fade and ESR evolution of Maxwell BCAP0010 P270 and Nesscap EHSR 0010C0-002R7 during $75 \%$ discontinuous cycling.

The temperature measured on the cells during the cycling is shown in Figure 9. The average temperature is around $30^{\circ} \mathrm{C}$ with some minor variations. Due to the higher ESR of the Maxwell cells, they operate at slightly higher temperature than the Nesscap cells. Both cells show a similarly good performance during the cycling with a capacity fade of around $12 \%$ and an increase of ESR of $30 \%$. After 600'000 cycles.

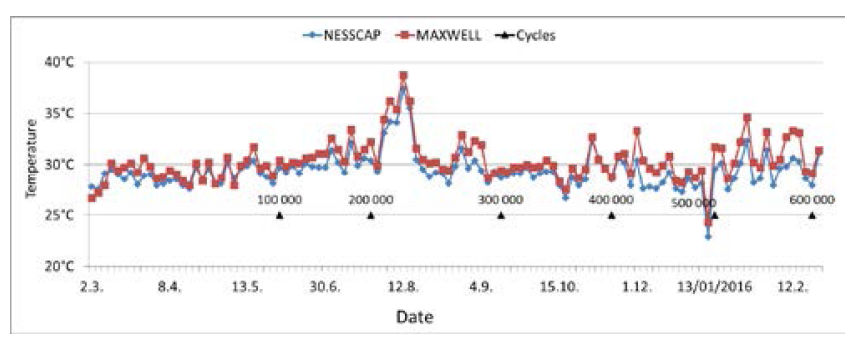

Figure 9: Temperature measured on the cells during the $75 \%$ discontinuous cycling test.

Finally, a life test under vacuum conditions was performed on the Nesscap cells.

The capacitance evolution is plotted on Figure 10 and compared with results under ambient pressure. 


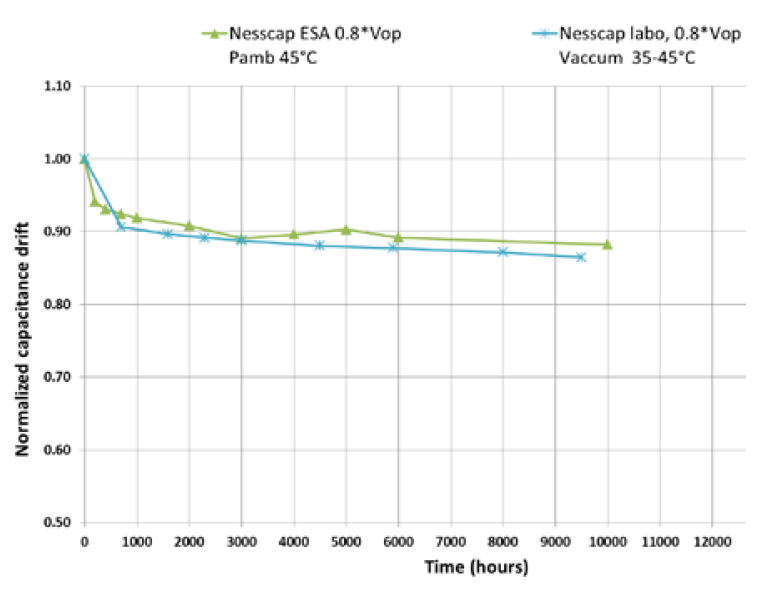

Figure 10: Capacity fade of Nesscap 10F in ambient pressure (green) and vacuum conditions.

The observed drifts are very similar to those observed in ambient pressure under the same conditions of voltage and temperature. This test campaign showed that the long term vacuum exposure does not deteriorate the performances of the Nesscap $10 \mathrm{~F}$ cells more strongly than in ambient pressure.

\subsection{Bank of Supercapacitor Tests}

\section{5s2p BOSC lifetest update}

BOSC2 (of initial activity as described in $[7,8]$ ), continued to cycle after it had completed 1.5 million cycles. After around 1.5 Million cycles corresponding to 290 full days of cycling the capacity fade was around $11 \%$ and the ESR increase around $16 \%$. The continued test results are shown in Figure 11. The capacity and the impedance at $1 \mathrm{kHz}$ are shown. There is a relatively pronounced capacity fade within the first 600'000 cycles, of which the rate decreases. The slope of the ESR curve decreases with increasing cycle number. After 4.5 million cycles the capacity has decreased by about $13 \%$ and the ESR has increased by around $36 \%$.

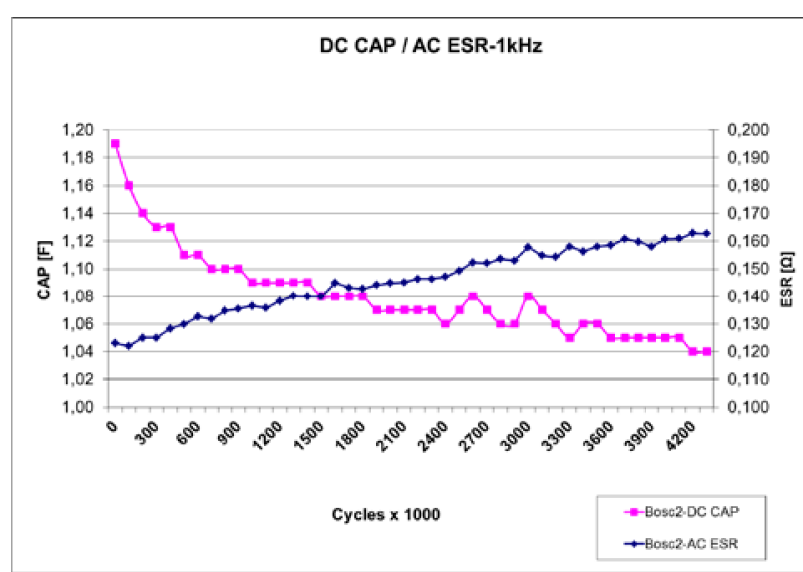

Figure 11: Capacity and ESR evolution during the life cycle test with the $15 s 2 p$ BOSC at $45^{\circ} \mathrm{C}$ with a discontinuous $75 \%$ energy cycling profile.

\section{4s1p BOSC life test results}

The results of the $4 \mathrm{~s} 1 \mathrm{p}$ BOSCs with different balancing resistors are shown in Figure 12.

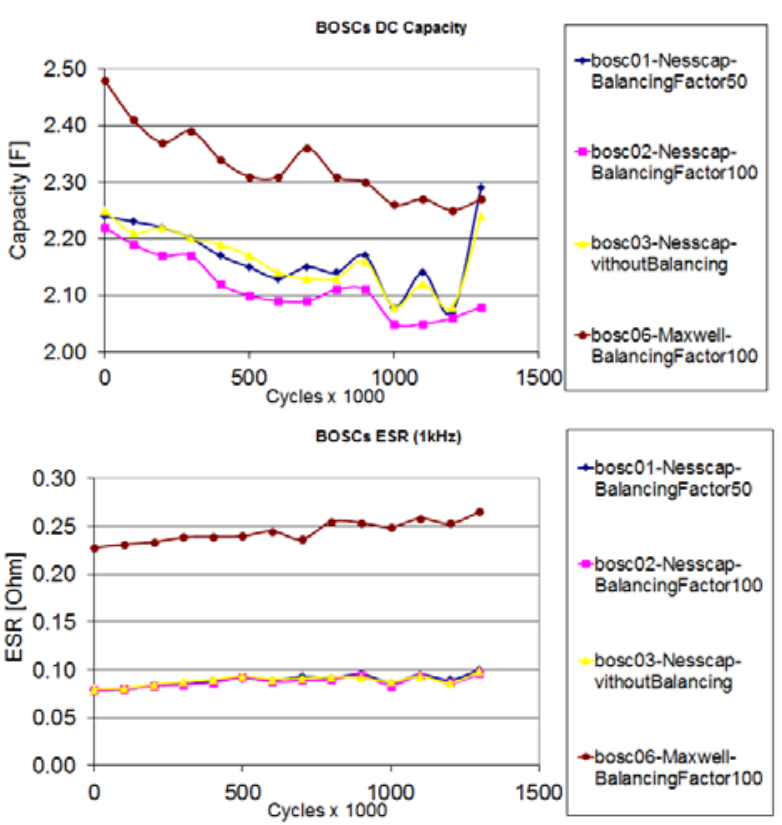

Figure 12: Overview of the Capacity and ESR evolution during the life cycle test of the 4sIp Nesscap and the Maxwell BOSCs with different balancing factors.

The capacity fade after around 1.2 million cycles is around $8 \%$ for the Nesscap BOSCs (average of all BOSCs), which is in excellent agreement with the results of the 15s $2 \mathrm{p}$ BOSC in Figure 11. Around 9\% were found for the Maxwell BOSC, which had a slightly higher capacity but also higher ESR as compared to the Nesscap BOSCs.

In Figure 13 the performance of the Cap-XX BOSC is shown. After around 700'000 cycles this BOSC failed due to the failure of cell 2 . It seems the negative contact of the cell became highly resistive by disconnection due to a venting event. The reason for this failure is not fully clear. 


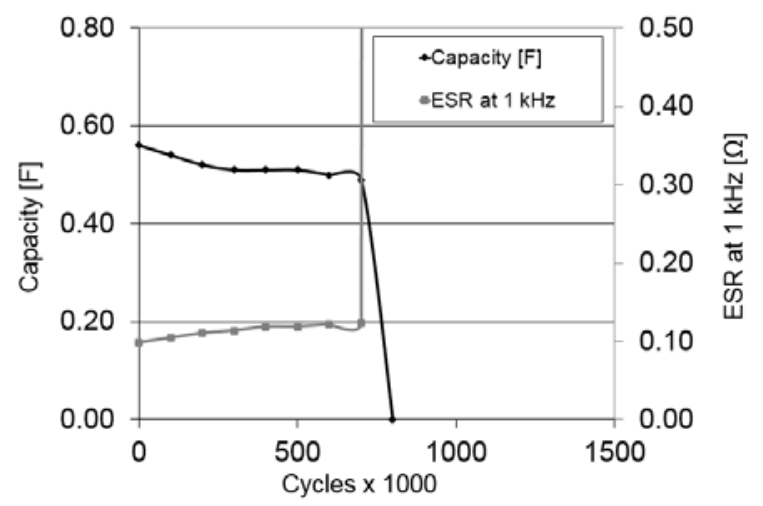

Figure 13: Capacity and ESR evolution during the life cycle test the 4s 1p Cap-XX BOSC with a balancing factor of 100.

\section{Balancing performance}

The individual cell voltage measurements of BOSC01 are shown in Figure 14. At the beginning of the test the cells are unbalanced but they quickly equilibrate. The difference between the maximum and minimum cell voltage (Delta Vmax) becomes quite constant and remains at a value of around $10 \mathrm{mV}$. The balancing therefore works as it should with a balancing factor of around 50 .

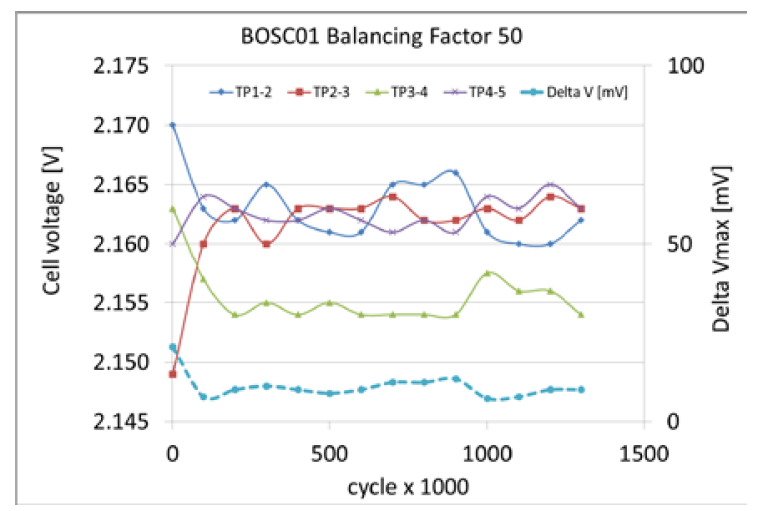

Figure 14: Individual cell voltages and Delta Vmax (right axis) of BOSC01 with balancing factor of 50.

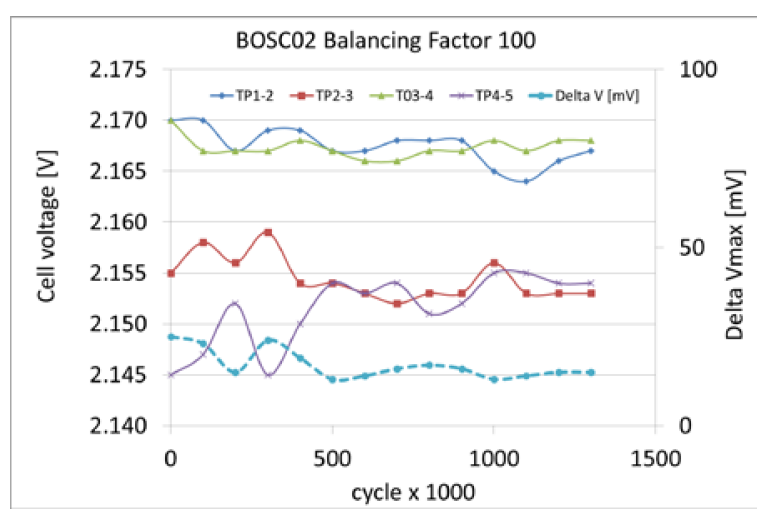

Figure 15: Individual cell voltages and Delta Vmax (right axis) of BOSC02 with balancing factor of 100.

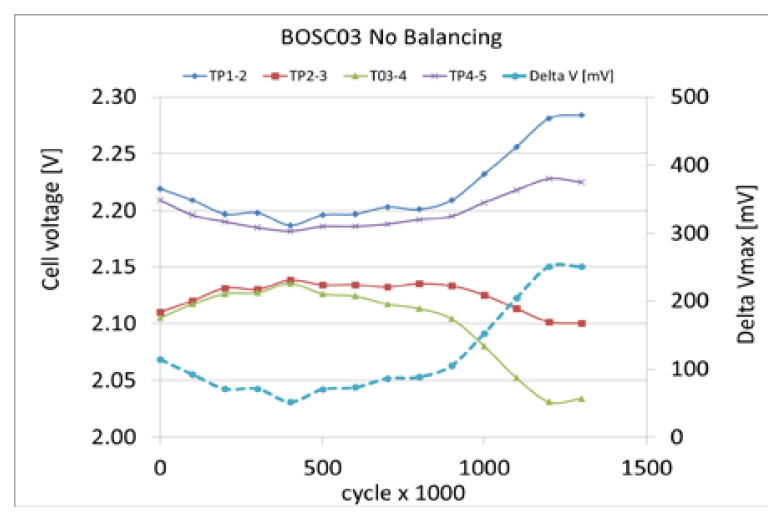

Figure 16: Individual cell voltages and Delta Vmax (right axis) of $\mathrm{BOSCO} 3$ without cell balancing.

With balancing factor of 100 the BOSC performed as shown in Figure 15. The difference of maximum and minimum cell voltages was around $15 \mathrm{mV}$ and stayed constant.

In contrast the BOSC without balancing shows diverging cell voltages as shown in Figure 16. A suboptimal life time can be expected with this pronounced imbalance of cell voltages.

After the tests the BOSCs were disassembled and each cell characterised. The measured capacities and ESRs were compared to the initial values. It seems that the 
presence of a balancing system allowed for a more homogeneous capacity fading of each cell in the BOSC as can be seen in Figure 17. Without balancing the average capacity fade was higher and the spread of the cells individual capacity fade was much larger at the end of the cycling test. No clear influence of the balancing on the ESR evolution could be seen.
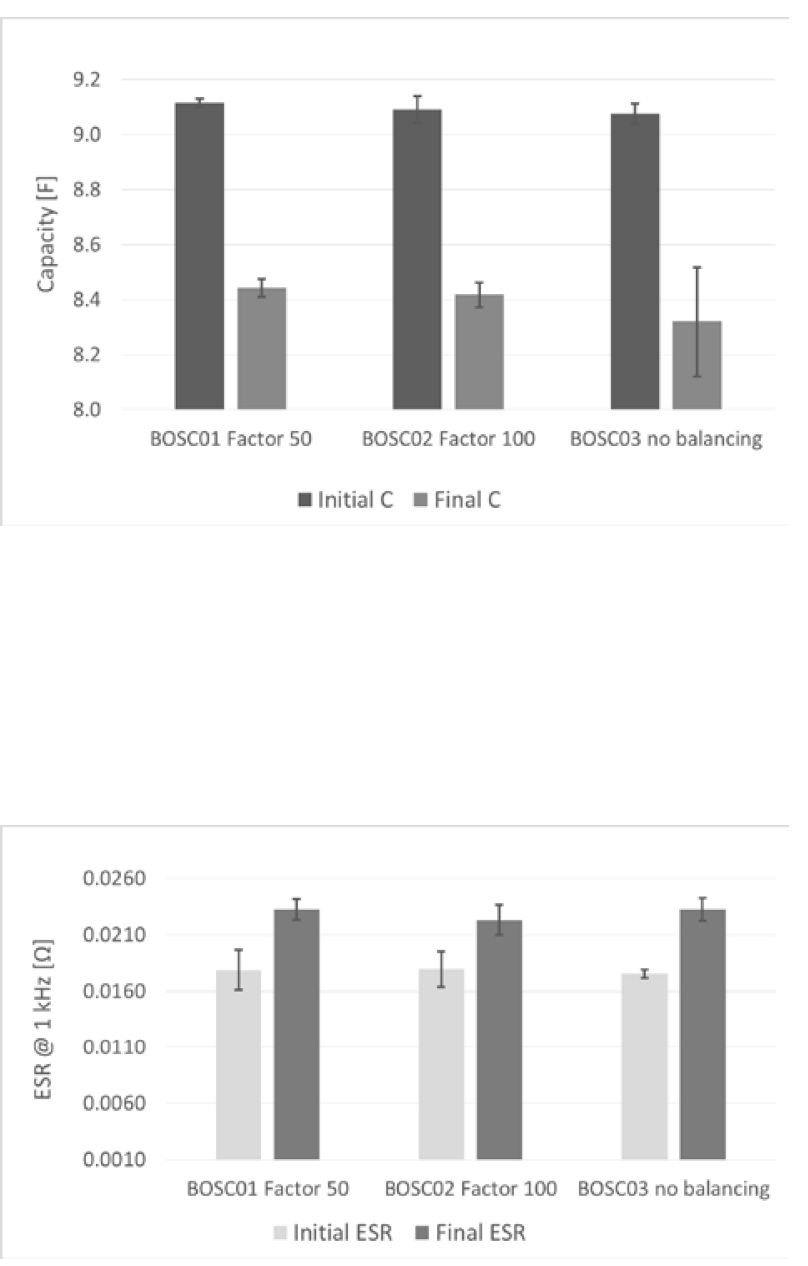

Figure 17: Average cell Capacities and ESRs before and after the cycling tests for BOSC01, BOSCO2 and BOSC03 without cell balancing.

The cell voltages of BOSC06, which was based on Maxwell cells is shown in Figure 18. The voltages of cell 1 and cell 3 showed a slightly increasing trend whereas the voltage of cell 4 decreased during the cycling. Cell 2 showed a more or less stable voltage. As compared to BOSC01 and BOSC02 Delta Vmax is about three times higher $(40 \mathrm{mV})$, possibly due to the higher internal resistance of the Maxwell cells.

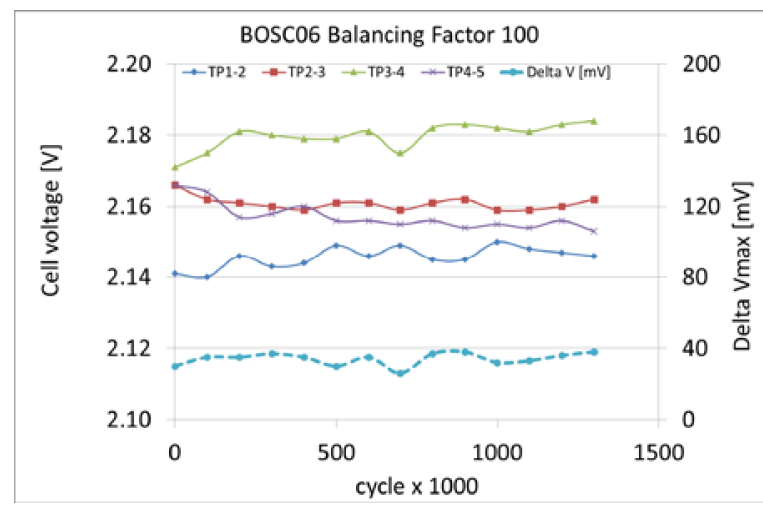

Figure 18: Individual cell voltages and Delta Vmax (right axis) of BOSC06 with Maxwell cells with a balancing factor of 100.

In Figure 19 the individual cell voltages of BOSC07 based on the Cap-XX cells are shown. There were pronounced changes in the cell voltages during the cycling. The cell that failed after around 700'000 cycles was the one with the lowest cell voltage (cell 2).

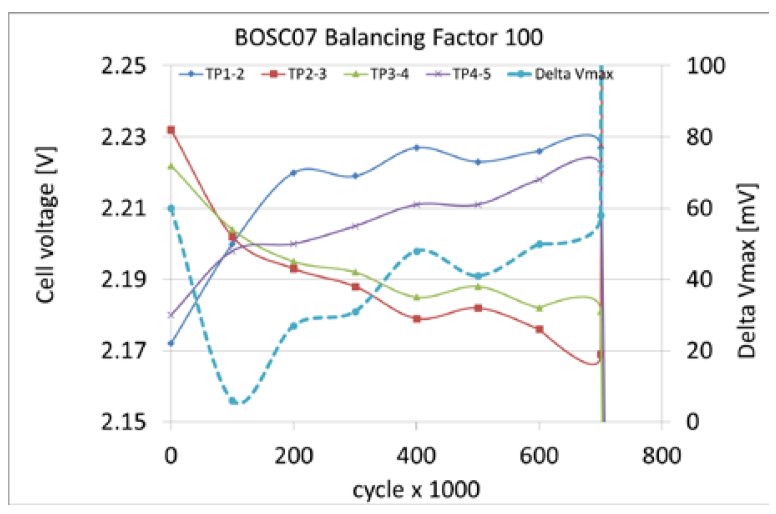

Figure 19: Individual cell voltages and Delta Vmax (right axis) of BOSCO7 with Cap-XX cells with a balancing factor of 100. 


\section{CONCLUSIONS AND FUTURE WORK}

The Nesscap and Maxwell supercapacitor cells are suitable for space applications if they are derated to $0.85 \cdot \mathrm{Vop}$ and operated at a maximum temperature of $45^{\circ} \mathrm{C}$. On BOSC level, more than 4 Million cycles could be demonstrated at these conditions. Based on the results of the $4 \mathrm{~s} 1 \mathrm{p}$ BOSCs a balancing system seems to be mandatory to guarantee a sufficiently long lifetime. The balancing system allows to equally distribute the aging of a BOSC to the individual cells. It is not trivial to find a correlation between the individual cell characteristics and the balancing status during the cycling tests. This would be an interesting topic for future investigations.

The benefits of the activity are numerous: confirmation of ageing models to extrapolate BOSC End-of-Life performances to 10 or 15 years, a first indication of the balancing factor and design to be applied and demonstration of vacuum performance.

From those results, the Nesscap cell is preferred when compared to Maxwell because of the low ESR, superior cycling performances, vacuum test demonstration and the manufacturer agreement for 5 years production continuity and traceability. Thanks to those activities, Maxwell could be identified as a credible back-up solution for the Nesscap cells.

All those complementary results have paved the way to a new project entitled "Generic Space Qualification of $10 \mathrm{~F}$ Nesscap Supercapacitors" aiming firstly to qualify Nesscap 10F supercapacitors for space applications and secondly to develop and qualify the associated BOSC (both shown in Figure 20). A generic and modular unit including components parallel/series arrangements compatible with several space systems is envisaged.
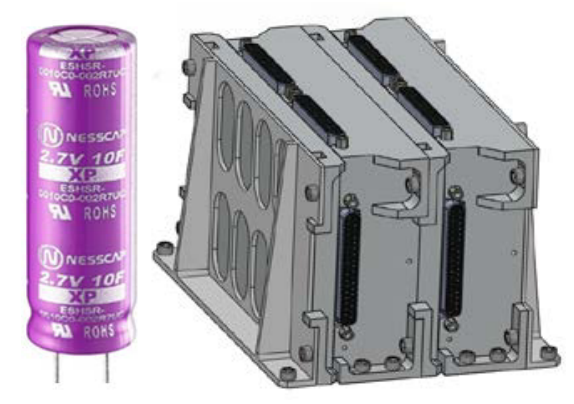

Figure 20: Nesscap ESHSR-0010C0-002R7UC supercapacitor under qualification and modular BOSC under development.

\section{ACKNOWLEDGEMENTS}

This research activity was conducted under ARTES 5.1 program element funded by the European Space Agency (Evaluation of Supercapacitors and Impact at System Level (4000105661/12/NL/NR). The view expressed herein can in no way be taken to reflect the official opinion of the European Space Agency.

\section{REFERENCES}

1. B.E. Conway, Electrochemical Super capacitors Scientific Fundamentals and Technological Applications, Kluwer and Plenum, New York, USA, 1999.

2. J.R. Miller, "CAPACITORS, Overview", Encyclopedia of Electrochemical Power Sources, Elsevier, Amsterdam, 2009, 587-599.

3. V. Gineste, D. Loup, P. Mattesco, N. Neugnot, "Preliminary Evaluation of Commercial Supercapacitors for Space Applications", 9th European Space Power Conference, Saint Raphael, France, 2011.

4. T. Shimizu, C. Underwood, "Super-capacitor energy storage for micro-satellites: Feasibility and potential mission applications", Acta Astronautica, 85, 138-154, 2013.

5. P. Kurzweil, "CAPACITORS, Electrochemical Double-Layer Capacitors", Encyclopedia of Electrochemical Power Sources, Elsevier, Amsterdam, 2009, 607-633.

6. H. Gualous, R. Gallay, M. Al Sakka, A. Oukaour, B. Tala-Ighil, B. Boudart, "Calendar and cycling ageing of activated carbon supercapacitor for automotive application", Microelectronics Reliability, 52, 24772481, 2012.

7. "ESA contract $n^{\circ} 1-6859 / 11 / \mathrm{NL} / \mathrm{NR}$ "Evaluation of supercapacitors and impacts at system level" including CCN1 and CCN2",

8. B. Buergler, E. Simon, P. Vasina, D. Latif, L. Diblik, V. Gineste, M. Simcak, "Design, Construction and Test of a Supecapacitor Bank for Space Applications" 10th European Space Power Conference, ESA, Noordwijkerhout, The Netherlands, 2014.

9. V. Sedlakova, J. Sikula, J. Majzner, P. Sedlak, T. Kuparowitz, B. Buergler, P. Vasina, "Supercapacitor equivalent electrical circuit model based on charges redistribution by diffusion", Journal of Power Sources, 286, 58-65, 2015. 